Article

\title{
Optimal Designs for Carry Over Effects the Case of Two Treatment and Four Periods
}

\author{
Miltiadis S. Chalikias
}

Department of Accounting and Finance, School of Business, Economics and Social Sciences, University of West Attica, 12244 Egaleo, Greece; mchalik@uniwa.gr or mchalikias@hotmail.com

Received: 23 September 2019; Accepted: 23 November 2019; Published: 3 December 2019

check for updates

\begin{abstract}
The optimal cross-over experimental designs are derived in experiments with two treatments, four periods, and an experimental unit. The results are given for the values $n=0 \bmod 4,1 \bmod 4,2 \bmod 4$ and $3 \bmod 4$. The criterion being the minimization of the variance of the estimated carry over effect.
\end{abstract}

Keywords: cross over designs; optimality; carry over effects

\section{Introduction}

The first usage of crossover experimental designs was in agriculture. In 1864 Lawes and Gilbert [1] introduced an experiment with two different treatments in the same agricultural parcel, this type of experiment has proven reliable to date. The provision of different treatments to the same experimental unit was not limited to only experiments in agriculture, it has been applied in Medicine and other scientific fields.

The majority of the experimenters search for optimal designs concerning the estimation of the parameters of a proposed model [2-10].

In crossover (or repeated measurement) designs, a sequence of treatments is applied to each experimental unit (e.u.) and one treatment is applied in each period [2]. In these designs either the estimation of the direct effect of the treatment, which is the effect of the treatment when being applied in the exam period, or the estimation of carry over, which is the effect of the treatment applied in the period before the examined one (i.e., period), can be of interest.

In the case of two treatments, $\mathrm{A}$ and $\mathrm{B}$, the carry over $\delta_{\mathrm{A}}$ and $\delta_{\mathrm{B}}$ can be estimated. In this paper, the parameter of interest is the difference of carry over effects $\delta=\delta_{\mathrm{A}}-\delta_{\mathrm{B}}$.

In the case of optimal designs with two treatments, the model introduced by Hedayat and Afsarinejad [10] is used with slight changes and optimal designs of direct and carry over for two periods were found in [11]. Kounias and Chalikias have presented the case of three periods for direct effects and for carry over effects in [12] and for a different model with treatment period interaction for direct effect in [13]. For the case of four periods and direct effects the optimal designs are available in [14]. In our case what will specified is the case of carry over effects in two treatments A, B, and four periods.

\section{Methodology}

\subsection{The Model}

For the case of four periods there are 16 sequences of treatments 


\begin{tabular}{cccccccc|cccccccc}
$A$ & $B$ & $A$ & $B$ & $A$ & $B$ & $A$ & $B$ & $A$ & $B$ & $A$ & $B$ & $A$ & $B$ & $A$ & $B$ \\
$A$ & $A$ & $B$ & $B$ & $A$ & $A$ & $B$ & $B$ & $A$ & $A$ & $B$ & $B$ & $A$ & $A$ & $B$ & $B$ \\
$A$ & $A$ & $A$ & $A$ & $B$ & $B$ & $B$ & $B$ & $A$ & $A$ & $A$ & $A$ & $B$ & $B$ & $B$ & $B$ \\
$A$ & $A$ & $A$ & $A$ & $A$ & $A$ & $A$ & $A$ & $B$ & $B$ & $B$ & $B$ & $B$ & $B$ & $B$ & $B$ \\
$u_{0}$ & $u_{1}$ & $u_{2}$ & $u_{3}$ & $u_{4}$ & $u_{5}$ & $u_{6}$ & $u_{7}$ & $u_{8}$ & $u_{9}$ & $u_{10}$ & $u_{11}$ & $u_{12}$ & $u_{13}$ & $u_{14}$ & $u_{15}$
\end{tabular}

where $u_{i}, i=0,1, \ldots, 15$ is the number of experimental units that received the $\mathrm{i}$-th sequence of treatments so $u_{0}+u_{1}+\cdots+u_{15}=n$, and all e.u.s are $n$.

The model is analogous to the cases of $[15,16]$ :

$$
y_{i j k}=\mu+\tau_{h}+\pi_{j}+\delta_{i, j-1}+\gamma_{i}+e_{i j k}
$$

where $j$ corresponds to the $j$-th period, $j=1,2,3,4$;

$i$ refers to the $i$-th sequence, $i=0,1, . .15$;

$k$ refers to the unit $k=1,2, \ldots, n$;

$h=A, B \tau_{\mathrm{A}}, \tau_{\mathrm{B}}$ are direct effects of treatments $\mathrm{A}$ and $\mathrm{B}$;

$\pi_{j}$ : is the effect of the $j$-th period;

$\delta_{\mathrm{A}}, \delta_{\mathrm{B}}$ : are the residual effects of $\mathrm{A}$ and $\mathrm{B}$;

$\gamma_{i}$ : is the effect of the $i$-th sequence; and

$e_{i j k}$ : independent of the errors normally distributed.

The errors $e_{i j k}$ are assumed to be independent between sequences and within sequences.

The above model (in overparameterized form) is written:

$$
\boldsymbol{\Upsilon}=\tau_{\mathrm{A}} \tau_{\mathrm{A}}+\tau_{\mathrm{B}} \boldsymbol{\tau}_{\mathrm{B}}+\delta_{\mathrm{A}} \boldsymbol{\delta}_{\mathrm{A}}+\delta_{\mathrm{B}} \boldsymbol{\delta}_{\mathrm{B}}+\pi_{1} \pi_{1}+\cdots+\pi_{4} \pi_{4}++\gamma_{0} \gamma_{0}+\cdots+\gamma_{15} \gamma_{15}+\mathbf{e}
$$

and $\boldsymbol{\Upsilon}, \boldsymbol{\tau}_{\mathrm{A}}, \boldsymbol{\tau}_{\mathrm{B}}, \boldsymbol{\delta}_{\mathrm{A}}, \boldsymbol{\delta}_{\mathrm{B}}, \boldsymbol{\pi}_{1}, \cdots \pi_{4}, \gamma_{0}, \cdots \gamma_{15}$, e are $1 \times 4 n$ vectors; the direct effect vector is 1 if the treatment is $\mathrm{A}$, and zero if it is $\mathrm{B}$. For example, for the sequence $\mathrm{ABB} \ldots, \boldsymbol{\tau}_{\mathrm{A}}=\left[\begin{array}{c}1 \\ 0 \\ 0 \\ \vdots\end{array}\right]$ is analogous to $\boldsymbol{\tau}_{B}=\left[\begin{array}{c}0 \\ 1 \\ 1 \\ \vdots\end{array}\right]$, $\boldsymbol{\delta}_{\mathrm{A}}=\left[\begin{array}{c}0 \\ 1 \\ 0 \\ \vdots\end{array}\right]$ and, in the same way, $\boldsymbol{\delta}_{\mathrm{B}}, \boldsymbol{\pi}_{i}$ and $\gamma_{i}$ are defined so that $\boldsymbol{\tau}_{\mathrm{A}}+\boldsymbol{\tau}_{\mathrm{B}}=1_{4 \mathbf{n}}, \boldsymbol{\delta}_{\mathrm{A}}+\boldsymbol{\delta}_{\mathrm{B}}+\boldsymbol{\pi}_{1}=$ $1_{4 \mathbf{n}}$, and $\pi_{1}+\pi_{2}+\pi_{3}+\pi_{4}=1_{4 \mathbf{n}}$. Also, 1 when the $i$ th unit is employed, and 0 elsewhere, so $\gamma_{0}+\gamma_{1}+\gamma_{2}+\ldots+\gamma_{15}=1_{4 \mathbf{n}}$. So, in Equation (2) there are linearly dependent vectors.

In a vector form:

$$
\boldsymbol{\Upsilon}=\mathbf{X b}+\mathbf{e} \Leftrightarrow \mathbf{Y}=\left(\begin{array}{ll}
\mathbf{X}_{1} & \mathbf{X}_{2}
\end{array}\right)\left(\begin{array}{l}
\mathbf{b}_{1} \\
\mathbf{b}_{2}
\end{array}\right)+\mathbf{e}
$$

where $\mathbf{Y}$ is $(4 n) \times 1, \mathbf{b}$ is $\mathbf{s} \times 1, \mathbf{X}$ is $(4 n) \times s$, $\mathbf{e}$ is $(4 n) \times 1$, and $s$ is the number of unknown parameters. We write $\mathbf{b}=\left(\begin{array}{ll}\mathbf{b}_{1} & \mathbf{b}_{2}\end{array}\right)$, where $\mathbf{b}_{1}$ is the vector of the $\mathbf{r}$ parameters of interest, and $\mathbf{b}_{2}$ is the vector of the s-r remaining parameters.

In our case $r=1$ and as it is referred the parameter of interest for the difference of the carry over effects, $\delta=\delta_{\mathrm{A}}-\delta_{\mathrm{B}}$ which is an estimable parameter as shown in the Proposition 2

Proposition 1. If $\mu, \pi_{1}, \pi_{2}, \pi_{3}, \pi_{4}, \tau_{A}, \tau_{B}, \gamma_{0}, \gamma_{1}, \ldots, \gamma_{15}$ are the vectors of $\mathbf{X}_{2}$ which corresponds to the parameters $\mu, \pi_{1}, \pi_{2}, \pi_{3}, \pi_{4}, \tau_{\mathrm{A}}, \tau_{\mathrm{B}}, \gamma_{0}, \gamma_{1}, \ldots, \gamma_{15}$, the vectors, $\pi_{1}, \pi_{2}, \pi_{3}, \tau=\tau_{\mathrm{A}}-\tau_{\mathrm{B}}, \gamma_{0}, \gamma_{1}, \ldots, \gamma_{15}$, are a base of the linear space $\mathrm{R}\left(\mathbf{X}_{2}\right)$. 
Proof. If we replace $\delta_{A}, \delta_{B}$ with $\delta_{A}+\delta_{B}, \delta_{A}-\delta_{B}$ the linear space $\mathrm{R}\left(\mathrm{X}_{2}\right)$ not change. Moreover we observe that $1_{4 n}=\mu=\pi_{1}+\delta_{\mathrm{A}}+\delta_{\mathrm{B}}$, and $\gamma_{0}+\gamma_{1}+\cdots+\gamma_{15}=1_{4 \mathrm{n}}$, so $\mu$ can be omitted and $\tau_{\mathrm{A}}+\tau_{\mathrm{B}}$ can be replaced with $\gamma_{15}$.

Corollary 1. If with $\tilde{\mathbf{X}}_{2}$ we note the space of $\boldsymbol{\pi}_{1}, \boldsymbol{\pi}_{2}, \boldsymbol{\pi}_{3}, \tau=\tau_{\mathrm{A}}-\tau_{\mathrm{B}}, \gamma_{0}, \gamma_{1}, \ldots, \gamma_{15}$, then $\mathrm{R}\left(\mathbf{X}_{2}\right)=\mathrm{R}\left(\tilde{\mathbf{X}}_{2}\right)$.

$$
\mathbf{X}_{1}=\left[\begin{array}{c}
\left.\mathbf{X}_{10}\right\} u_{0} \\
\left.\mathbf{X}_{11}\right\} u_{1} \\
\vdots \\
\left.\mathbf{X}_{115}\right\} u_{15}
\end{array}\right], \mathbf{X}_{2}=\left[\begin{array}{cccccc}
\mathbf{X}_{20} & 1_{4} & 0 & \cdots & 0 & 0 \\
\mathbf{X}_{21} & 0 & 1_{4} & \cdots & 0 & 0 \\
\vdots & \vdots & \vdots & \cdots & \vdots & \vdots \\
\mathbf{X}_{214} & 0 & 0 & \cdots & 0 & 1_{4} \\
\mathbf{X}_{215} & 0 & 0 & 0 & 0 & 0
\end{array}\right]
$$

Matrixes $\mathbf{X}_{1 i}, \mathbf{X}_{2 i}, i=1,2 . .15$ are available in Appendix A.

\subsection{Estimation of the Carry Over Effects}

Using the Best Linear Unbiased Estimators (BLUE) $\delta_{\mathrm{A}}, \delta_{\mathrm{B}}[16,17]$ we have:

$$
\left(\mathbf{X}_{1}^{\mathrm{T}} \mathbf{X}_{1}-\mathbf{X}_{1}^{\mathrm{T}} \mathbf{P} \mathbf{X}_{1}\right)\left(\begin{array}{c}
\hat{\delta}_{\mathrm{A}} \\
\hat{\delta}_{\mathrm{B}}
\end{array}\right)=\mathbf{X}_{1}^{\mathrm{T}}(\mathbf{I}-\mathbf{P}) \mathbf{Y}
$$

where $\mathbf{X}_{1}=\left[\delta_{\mathrm{A}}-\boldsymbol{\delta}_{\mathrm{B}}\right], \mathbf{X}_{2}=\left[\boldsymbol{\mu}, \boldsymbol{\pi}_{1}, \boldsymbol{\pi}_{2}, \boldsymbol{\pi}_{3}, \tau_{\mathrm{A}}, \boldsymbol{\gamma}_{1}, \gamma_{2}, \ldots, \boldsymbol{\gamma}_{14}\right]$, and $\mathbf{P}=\mathbf{X}_{2}\left(\mathbf{X}_{2}^{\mathrm{T}} \mathbf{X}_{2}\right)^{-1} \mathbf{X}_{2}^{\mathrm{T}}$ is the $(4 n) \times$ $(4 n)$ projection matrix in $R\left(\mathbf{X}_{2}\right)$.

Proposition 2. The carry over effects $\delta_{\mathrm{A}}, \delta_{\mathrm{B}}, \delta_{\mathrm{A}}+\delta_{\mathrm{B}}$ cannot be estimated, but the difference can be estimated $\delta_{\mathrm{A}}-\delta_{\mathrm{B}}$ with $\operatorname{var}\left(\hat{\delta}_{\mathrm{A}}-\hat{\delta}_{\mathrm{B}}\right)=\sigma^{2} G^{-1}, G=\delta_{\mathrm{A}}^{T}(I-P) \delta_{\mathrm{A}}=\delta_{\mathrm{B}}^{T}(I-P) \delta_{\mathrm{B}}=-\delta_{\mathrm{A}}^{T}(I-P) \delta_{\mathrm{B}}$.

Proof. We observe that $\boldsymbol{\delta}_{\mathrm{A}}+\boldsymbol{\delta}_{\mathrm{B}}+\boldsymbol{\pi}_{1}=1$, so $\left(\boldsymbol{\delta}_{\mathrm{A}}+\boldsymbol{\delta}_{\mathrm{B}}\right) \in R\left(\mathbf{X}_{2}\right)$ and $(\mathbf{I}-\mathbf{P})\left(\boldsymbol{\delta}_{\mathrm{A}}+\boldsymbol{\delta}_{\mathrm{B}}\right)=0$, then $\boldsymbol{\delta}_{\mathrm{A}}^{T}(\mathbf{I}-\mathbf{P}) \boldsymbol{\delta}_{\mathrm{A}}=-\boldsymbol{\delta}_{A}^{\tau}(I-P) \boldsymbol{\delta}_{\mathrm{B}}=\boldsymbol{\delta}_{B}^{\tau}(I-P) \boldsymbol{\delta}_{\mathrm{B}}=G$.

We have,

$$
\left[\begin{array}{ll}
\boldsymbol{\delta}_{\mathrm{A}}^{\mathrm{T}}(\mathbf{I}-\mathbf{P}) \boldsymbol{\delta}_{\mathrm{A}} & \boldsymbol{\delta}_{\mathrm{A}}^{\mathrm{T}}(\mathbf{I}-\mathbf{P}) \boldsymbol{\delta}_{\mathrm{B}} \\
\boldsymbol{\delta}_{\mathrm{B}}^{\mathrm{T}}(\mathbf{I}-\mathbf{P}) \boldsymbol{\delta}_{\mathrm{A}} & \boldsymbol{\delta}_{\mathrm{B}}^{\mathrm{T}}(\mathbf{I}-\mathbf{P}) \boldsymbol{\delta}_{\mathrm{B}}
\end{array}\right]\left[\begin{array}{c}
\hat{\delta}_{A} \\
\hat{\delta}_{\mathrm{B}}
\end{array}\right]=\left[\begin{array}{c}
G\left(\hat{\delta}_{A}-\hat{\delta}_{\mathrm{B}}\right) \\
-G\left(\hat{\delta}_{A}-\hat{\delta}_{\mathrm{B}}\right)
\end{array}\right]=\left[\begin{array}{c}
\boldsymbol{\delta}_{\mathrm{A}}^{\mathrm{T}}(\mathbf{I}-\mathbf{P}) \mathbf{Y} \\
\boldsymbol{\delta}_{B}^{\mathrm{T}}(\mathbf{I}-\mathbf{P}) \mathbf{Y}
\end{array}\right]
$$

where $\operatorname{var}\left(\hat{\delta}_{\mathrm{A}}-\hat{\delta}_{\mathrm{B}}\right)=\sigma^{2} G^{-1}$. So $\delta, \delta, \delta+\delta$ cannot be estimate, on the contrary $\delta-\delta$ can be estimate.

Proposition 3. $Q=\mathbf{X}_{1}^{\mathrm{T}}\left(\mathbf{I}_{4 \mathbf{n}}-\mathbf{P}\right) \mathbf{X}_{1}=\mathbf{X}_{1}^{\mathrm{T}}\left(\mathbf{I}_{4 \mathbf{n}}-\tilde{\mathbf{P}}\right) \mathbf{X}_{1}$ where $\tilde{\mathbf{P}}=\tilde{\mathbf{X}}_{2}\left(\tilde{\mathbf{X}}_{2} \tilde{\mathbf{X}}_{2}\right)^{-1} \tilde{\mathbf{X}}_{2}^{\mathbf{T}}$.

Proof. $\mathbf{P} \mathbf{X}_{1}$ is the orthogonal projection of $\mathbf{X}_{1}$ to the linear space of $R\left(\mathbf{X}_{2}\right)$. Nevertheless $R\left(\mathbf{X}_{2}\right)=R\left(\tilde{\mathbf{X}}_{2}\right)$, so $\mathbf{P X}_{1}=\tilde{\mathbf{P}} \mathbf{X}_{1}$ and $\mathbf{X}_{1}^{\mathbf{T}}\left(\mathbf{I}_{4 \mathbf{n}}-\mathbf{P}\right) \mathbf{X}_{1}=\mathbf{X}_{1}^{\mathbf{T}}\left(\mathbf{I}_{4 \mathbf{n}}-\tilde{\mathbf{P}}\right) \mathbf{X}_{1}$.

2.3. Calulation of $\boldsymbol{X}_{1}^{T}\left(\boldsymbol{I}_{4 n}-\tilde{\boldsymbol{P}}\right) \boldsymbol{X}_{1}$

So we need to calculate the minimum of the $\mathbf{X}_{1}^{\mathrm{T}}\left(\mathbf{I}_{4 \mathbf{n}}-\tilde{\mathbf{P}}\right) \mathbf{X}_{1}$.

Proposition 4. If $\mathbf{w}=\left(x_{1}, x_{2}, x_{3}, x_{4}, z_{0}, z_{1}, \ldots, z_{15}\right)^{T}$, then

$$
\mathbf{X}_{1}^{\mathbf{T}}\left(\mathbf{I}_{4 \mathbf{n}}-\tilde{\mathbf{P}}\right) \mathbf{X}_{1}=\min _{\mathbf{w}}\left(\mathbf{X}_{1}-\tilde{\mathbf{X}}_{2} \mathbf{w}\right)^{T}\left(\mathbf{X}_{1}-\tilde{\mathbf{X}}_{2} \mathbf{w}\right)
$$


Proof. If $T$ a point of the linear space $\mathbf{R}\left(\tilde{\mathbf{X}}_{2}\right)$ with $T=\tilde{\mathbf{X}}_{2} \mathbf{w}$, then $\left(\mathbf{X}_{1}-\tilde{\mathbf{X}}_{2} \mathbf{w}\right)^{\mathbf{T}}\left(\mathbf{X}_{1}-\tilde{\mathbf{X}}_{2} \mathbf{w}\right)$ is the square of the distance of $\mathrm{T}$ from the space $\mathbf{X}_{1}$, so $\mathbf{X}_{1}^{\mathrm{T}}\left(\mathbf{I}_{4 \mathbf{n}}-\tilde{\mathbf{P}}\right) \mathbf{X}_{1}=\min _{w}\left(\mathbf{X}_{1}-\mathbf{X}_{2} \mathbf{w}\right)^{\mathbf{T}}\left(\mathbf{X}_{1}-\mathbf{X}_{2} \mathbf{w}\right)$.

$$
\begin{aligned}
& \text { If } F(\mathbf{x}, \mathbf{z})=\left(\mathbf{X}_{1}-\tilde{\mathbf{X}}_{2} \mathbf{w}\right)^{\mathbf{T}}\left(\mathbf{X}_{1}-\tilde{\mathbf{X}}_{2} \mathbf{w}\right) \text {, then } F(\mathbf{x}, \mathbf{z})=\mathbf{X}_{1}^{\mathrm{T}} \mathbf{X}_{1}-2 \mathbf{X}_{1}^{\mathrm{T}} \tilde{\mathbf{X}}_{2} \mathbf{w}+\mathbf{w}^{\mathbf{T}} \tilde{\mathbf{X}}_{2}^{\mathbf{T}} \tilde{\mathbf{X}}_{2} \mathbf{w} \text {, and } \\
& \mathbf{X}_{1}^{\mathrm{T}} \mathbf{X}_{1}=u_{0} \mathbf{X}_{10}^{\mathrm{T}} \mathbf{X}_{10}+u_{1} \mathbf{X}_{11}^{\mathrm{T}} \mathbf{X}_{11}+\cdots+u_{15} \mathbf{X}_{1,15}^{\mathrm{T}} \mathbf{X}_{1,15} \\
& \mathbf{X}_{1}^{\mathbf{T}} \tilde{\mathbf{X}}_{2} \mathbf{w}=u_{0} \mathbf{X}_{10}^{\mathrm{T}}\left(\tilde{\mathbf{X}}_{20}+z_{0} 1_{4}\right)+u_{1} \mathbf{X}_{11}^{\mathrm{T}}\left(\tilde{\mathbf{X}}_{21}+z_{1} 1_{4}\right)+\cdots \\
& +u_{15} \mathbf{X}_{1,15}^{\mathrm{T}}\left(\tilde{\mathbf{X}}_{2,15}+z_{15} 1_{4}\right) \\
& \mathbf{w}^{\mathbf{T}} \tilde{\mathbf{X}}_{2}^{\mathbf{T}} \tilde{\mathbf{X}}_{2} \mathbf{w}=\mathbf{u}_{0}\left[\tilde{\mathbf{X}}_{20}^{\mathbf{T}} \tilde{\mathbf{X}}_{20}+2 z_{0} \tilde{\mathbf{X}}_{20}^{\mathbf{T}} 1_{4}+z_{0}^{2} 1_{4}^{\mathbf{T}} 1_{4}\right] \\
& +\mathbf{u}_{1}\left[\tilde{\mathbf{X}}_{21}^{\mathbf{T}} \tilde{\mathbf{X}}_{21}+2 z_{1} \tilde{\mathbf{X}}_{21} 1_{4}+z_{1}^{2} 1_{4}^{\mathbf{T}} 1_{4}\right]+\cdots \\
& +\mathbf{u}_{15}\left[\tilde{\mathbf{X}}_{2,15}^{\mathbf{T}} \tilde{\mathbf{X}}_{2,15}+2 z_{15} \tilde{\mathbf{X}}_{2,15} 1_{4}+z_{15}^{2} 1_{4}^{\mathbf{T}} 1_{4}\right]
\end{aligned}
$$

In order to minimize $F(x, z)$, we have, $\min F(\mathbf{x}, \mathbf{z})=\min _{\mathbf{x}}\left(\min _{\mathbf{z}} F(\mathbf{x}, \mathbf{z})\right)$, so:

$$
\begin{array}{ccc}
\frac{\partial F(\mathbf{x}, \mathbf{z})}{\partial z_{0}}= & 2 u_{0}\left[-\mathbf{X}_{10}^{\mathbf{T}} 1_{4}+\tilde{\mathbf{X}}_{20}^{\mathbf{T}} 1_{4}+4 z_{0}\right]=0 \\
\vdots & \vdots & \vdots \\
\frac{\partial F(\mathbf{x}, \mathbf{z})}{\partial z_{15}}= & 2 u_{15}\left[-\mathbf{X}_{1,15}^{\mathbf{T}} 1_{4}+\tilde{\mathbf{X}}_{2,15}^{\mathbf{T}} 1_{4}+4 z_{15}\right]=0
\end{array} .
$$

If we replace the solution of $z_{0}, z_{1}, \ldots, z_{15}$ in $F(\mathbf{x}, \mathbf{z})$, we have:

$$
\begin{aligned}
& F(\mathbf{x})=u_{0}\left[\mathbf{X}_{10}^{\mathrm{T}} \mathbf{X}_{10}-2 \mathbf{X}_{10}^{\mathrm{T}} \tilde{\mathbf{X}}_{20}+\tilde{\mathbf{X}}_{20}^{\mathbf{T}} \mathbf{X}_{20}-\frac{\left(\mathbf{X}_{10}^{\mathrm{T}} 1_{4}-\tilde{\mathbf{X}}_{20} 1_{4}\right)^{2}}{4}\right]+\cdots \\
& +u_{15}\left[\mathbf{X}_{1,15}^{\mathrm{T}} \mathbf{X}_{1,15}-2 \mathbf{X}_{1,15}^{\mathrm{T}} \tilde{\mathbf{X}}_{2,15}+\tilde{\mathbf{X}}_{2,15}^{\mathbf{T}} \mathbf{X}_{2,15}-\frac{\left(\mathbf{X}_{1,15}^{\mathrm{T}} 1_{4}-\tilde{\mathbf{X}}_{2,15}{ }^{\mathrm{T}}{ }^{2}\right)^{2}}{4}\right]
\end{aligned}
$$

$F(x)$ is of the form

$$
F(\mathbf{x})=\frac{1}{4}\left[R-2 \tilde{\mathbf{q}}^{\mathbf{T}} \mathbf{x}+\mathbf{x}^{\mathbf{T}} \tilde{\mathbf{M}} \mathbf{x}\right]
$$

where $R=u_{0}\left[4 \mathbf{X}_{10}^{\mathrm{T}} \mathbf{X}_{10}-\left(\mathbf{X}_{10}^{\mathrm{T}} 1_{4}\right)^{2}\right]+u_{1}\left[4 \mathbf{X}_{11}^{\mathrm{T}} \mathbf{X}_{11}-\left(\mathbf{X}_{11}^{\mathrm{T}} 1_{4}\right)^{2}\right]+\cdots$

$$
+u_{15}\left[4 \mathbf{X}_{1,15}^{\mathbf{T}} \mathbf{X}_{1,15}-\left(\mathbf{X}_{1,15}^{\mathbf{T}} 1_{4}\right)^{2}\right]
$$




$$
\begin{aligned}
& \tilde{\mathbf{q}}^{T} \mathbf{x}=u_{0}\left[4 \mathbf{X}_{10}^{\mathbf{T}} \tilde{\mathbf{X}}_{20}-\left(\mathbf{X}_{10}^{\mathbf{T}} 1_{4}\right)\left(\tilde{\mathbf{X}}_{20}^{\mathbf{T}} 1_{4}\right)\right] \\
& +u_{1}\left[4 \mathbf{X}_{11}^{\mathbf{T}} \tilde{\mathbf{X}}_{21}-\left(\mathbf{X}_{11}^{\mathbf{T}} 1_{4}\right)\left(\tilde{\mathbf{X}}_{21}^{\mathbf{T}} 1_{4}\right)\right]+\cdots \\
& +u_{15}\left[4 \mathbf{X}_{1,15}^{\mathbf{T}} \tilde{\mathbf{X}}_{2,15}-\left(\mathbf{X}_{1,15}^{\mathbf{T}} 1_{4}\right)\left(\tilde{\mathbf{X}}_{2,15}^{\mathbf{T}} 1_{4}\right)\right]
\end{aligned}
$$

Equation (5) is a quadratic form of $x$ and the minimization is of the form:

$$
G=\min _{x} F(\mathbf{x})=\frac{1}{4}\left[R-\tilde{\mathbf{q}}^{\mathrm{T}} \tilde{\mathbf{M}}^{-1} \tilde{\mathbf{q}}\right]
$$

\section{Results}

\subsection{Calculation of $G$ in Case of four Periods and Carry Over Effects}

Using the above matrixes we have

$$
4 G=\left(\boldsymbol{\delta}_{\mathrm{A}}-\boldsymbol{\delta}_{\mathrm{B}}\right)^{\mathbf{T}}(\mathbf{I}-\mathbf{P})\left(\boldsymbol{\delta}_{\mathrm{A}}-\boldsymbol{\delta}_{\mathrm{B}}\right)
$$

where $\mathbf{X}_{1}=\delta_{\mathrm{A}}-\delta_{\mathrm{B}}$, then,

$$
\begin{aligned}
& X_{10}=X_{18}=\left[\begin{array}{l}
0 \\
1 \\
1 \\
1
\end{array}\right], X_{11}=X_{19}=\left[\begin{array}{c}
0 \\
-1 \\
1 \\
1
\end{array}\right], X_{12}=X_{1,10}=\left[\begin{array}{c}
0 \\
1 \\
-1 \\
1
\end{array}\right] \text {, } \\
& X_{13}=X_{1,11}=\left[\begin{array}{c}
0 \\
-1 \\
-1 \\
1
\end{array}\right], X_{14}=X_{1,12}=\left[\begin{array}{c}
0 \\
-1 \\
1 \\
-1
\end{array}\right], X_{15}=X_{1,13}=\left[\begin{array}{c}
0 \\
-1 \\
1 \\
-1
\end{array}\right] \text {, } \\
& X_{16}=X_{1,14}=\left[\begin{array}{c}
0 \\
1 \\
-1 \\
-1
\end{array}\right], X_{17}=X_{1,15}=\left[\begin{array}{c}
0 \\
-1 \\
-1 \\
-1
\end{array}\right] \\
& \mathbf{X}_{20}=\left[\begin{array}{c}
x_{1}+x_{4} \\
x_{2}+x_{4} \\
x_{3}+x_{4} \\
x_{4}
\end{array}\right], \quad \mathbf{X}_{21_{1}}=\left[\begin{array}{c}
x_{1}-x_{4} \\
x_{2}+x_{4} \\
x_{3}+x_{4} \\
x_{4}
\end{array}\right], \mathbf{X}_{22_{1}}=\left[\begin{array}{c}
x_{1}+x_{4} \\
x_{2}-x_{4} \\
x_{3}+x_{4} \\
x_{4}
\end{array}\right], \mathbf{X}_{23_{1}}=\left[\begin{array}{c}
x_{1}-x_{4} \\
x_{2}-x_{4} \\
x_{3}+x_{4} \\
x_{4}
\end{array}\right], \\
& X_{2,15-i}=2\left[\begin{array}{c}
x_{1} \\
x_{2} \\
x_{3} \\
0
\end{array}\right]-X_{2 i}, i=0,1, \ldots, 7, \mathbf{X}_{1 \mathbf{i}} \Leftrightarrow\left(\delta_{\mathrm{A}}-\delta_{\mathrm{B}}\right)_{i}, \iota=0,1, \ldots, 15
\end{aligned}
$$

Using the relations (6) we have $R=11 n-8\left(u_{0}+u_{7}+u_{8}+u_{15}\right)$

$$
q=\left[\begin{array}{l}
q_{1} \\
q_{2} \\
q_{3} \\
q_{4}
\end{array}\right], M=\left[\begin{array}{cccc}
3 n & -n & -n & m_{14} \\
-n & 3 n & -n & m_{24} \\
-n & -n & 3 n & m_{34} \\
m_{14} & m_{24} & m_{34} & m_{44}
\end{array}\right]
$$




$$
\begin{aligned}
q_{1}= & -3\left(u_{0}-u_{15}\right)-\left(u_{1}-u_{14}\right)-\left(u_{2}-u_{13}\right)+\left(u_{3}-u_{12}\right)-\left(u_{4}-u_{11}\right) \\
& +\left(u_{5}-u_{10}\right)+\left(u_{6}-u_{9}\right)+3\left(u_{7}-u_{8}\right) \\
q_{2}= & \left(u_{0}-u_{15}\right)-5\left(u_{1}-u_{14}\right)+3\left(u_{2}-u_{13}\right)-3\left(u_{3}-u_{12}\right)+3\left(u_{4}-u_{11}\right) \\
& -3\left(u_{5}-u_{10}\right)+5\left(u_{6}-u_{9}\right)-\left(u_{7}-u_{8}\right) \\
q_{3}= & \left(u_{0}-u_{15}\right)+3\left(u_{1}-u_{14}\right)-5\left(u_{2}-u_{13}\right)-3\left(u_{3}-u_{12}\right)+3\left(u_{4}-u_{11}\right) \\
& +5\left(u_{5}-u_{10}\right)-3\left(u_{6}-u_{9}\right)-\left(u_{7}-u_{8}\right) \\
q_{4}= & 2\left(u_{1}+u_{14}\right)-6\left(u_{2}+u_{13}\right)+4\left(u_{3}+u_{12}\right)-6\left(u_{4}+u_{11}\right)-12\left(u_{5}+u_{10}\right) \\
& -4\left(u_{6}+u_{9}\right)-2\left(u_{7}+u_{8}\right) \\
m_{14}= & -6\left(u_{1}-u_{14}\right)+2\left(u_{2}-u_{13}\right)-4\left(u_{3}-u_{12}\right)+2\left(u_{4}-u_{11}\right)-4\left(u_{5}-u_{10}\right)+4\left(u_{6}-u_{9}\right) \\
m_{24}= & 2\left(u_{1}-u_{14}\right)-6\left(u_{2}-u_{13}\right)-4\left(u_{3}-u_{12}\right)+2\left(u_{4}-u_{11}\right)+4\left(u_{5}-u_{10}\right)-4\left(u_{6}-u_{9}\right) \\
m_{34}= & 2\left(u_{1}-u_{14}\right)+2\left(u_{2}-u_{13}\right)+4\left(u_{3}-u_{12}\right)-6\left(u_{4}-u_{11}\right)-4\left(u_{5}-u_{10}\right)-4\left(u_{6}-u_{9}\right) \\
m_{44}= & 12\left(u_{1}+u_{14}\right)+12\left(u_{2}+u_{13}\right)+16\left(u_{3}+u_{12}\right)+12\left(u_{4}+u_{11}\right)+16\left(u_{5}+u_{10}\right) \\
& +16\left(u_{6}+u_{9}\right) .
\end{aligned}
$$

\subsection{Optimal Designs for Carry Over Effects}

The optimal designs minimize the variance $\operatorname{var}\left(\hat{\delta}_{\mathrm{A}}-\hat{\delta}_{\mathrm{B}}\right)=\sigma^{2} G^{-1}$ so have to maximize the quantity $R-\mathbf{q}^{\mathbf{T}} \mathbf{M}^{-1} \mathbf{q}$.

Proposition 5. In the case of four periods and in order to find the optimal designs for the carry over effects two cases of $n$ are examined:

(i) $n=0 \bmod 2: G^{*}=\max _{u_{i}} G=\frac{11 n}{16}$, and the solutions are, $u_{0}=u_{7}=u_{8}=u_{15}=0$,

$$
\begin{aligned}
& \left(u_{6}-u_{9}\right)-\left(u_{1}-u_{14}\right)=0 \\
& \left(u_{5}-u_{10}\right)-\left(u_{2}-u_{13}\right)=0 \\
& \left(u_{4}-u_{11}\right)-\left(u_{3}-u_{12}\right)=0 \\
& \left(u_{1}+u_{14}\right)-3\left(u_{2}+u_{13}\right)+2\left(u_{3}+u_{12}\right)-3\left(u_{4}+u_{11}\right)-6\left(u_{5}+u_{10}\right)-2\left(u_{6}+u_{9}\right)=0 . \\
& \left(u_{1}+u_{2}+u_{3}+u_{11}+u_{10}+u_{9}=\frac{n}{2}\right.
\end{aligned} .
$$

Some solutions which satisfy the above correlations are:

$(\alpha)$

$$
u_{1}=\frac{n}{6}, u_{14}=\frac{n}{6}+d, u_{3}=u_{4}=f, u_{9}=d \quad f+d=\frac{n}{3}, n=0 \bmod 6
$$

$u_{i}=0 \quad i \neq 1,3,4,9,14$

( $\beta)$

$u_{1}=u_{14}=\frac{3 n}{8}, u_{4}=u_{11}=\frac{n}{8}, n=0 \bmod 8$

$u_{i}=0 \quad i \neq 1,4,11,14$

$(\gamma)$

$u_{1}=u_{14}=\frac{2 n}{6}, u_{6}=u_{9}=\frac{n}{6}, n=0 \bmod 6$

$u_{i}=0 \quad i \neq 1,6,9,14$

$(\delta)$

$u_{3}-2=u_{12}=\frac{3 n}{10}-1, u_{4}-2=u_{11}=\frac{n}{5}-1, n=0 \bmod 10$

$u_{i}=0 \quad i \neq 3,4,11,12$

$(\varepsilon)$

$u_{1}=u_{14}=n-5 \beta, u_{2}=u_{13}=\beta, u_{3}=u_{12}=4 \beta-\frac{n}{2} \beta \in\left\{\frac{n}{8}, \ldots,\left[\frac{n}{5}\right]\right\}, n \geq 8$

$u_{i}=0 \quad i \neq 1,2,3,12,13,14$

$(\sigma \tau)$

$$
u_{1}=u_{3}=u_{4}=\frac{n+2}{7}, u_{9}=u_{11}=u_{12}=\frac{n-5}{7}, u_{14}=\frac{n+9}{7}, n=12 \bmod 14
$$

$u_{i}=0 i \neq 1,3,4,9,11,12,14$,

(द)

$u_{3}=u_{12}=\frac{n}{8}, u_{5}=u_{10}=\frac{3 n}{8}, n=0 \bmod 8$

$u_{i}=0 \quad i \neq 3,5,10,12$ 
( $)$

$u_{3}=u_{12}=\frac{n}{8}, u_{5}=u_{10}=\frac{3 n}{8}, n=0 \bmod 8$

$u_{i}=0 \quad i \neq 3,5,10,12$

( $\theta)$

$u_{3}=u_{12}=u_{6}=u_{9}=\frac{n}{4}, \quad n=0 \bmod 4$

$u_{i}=0 \quad i \neq 3,6,9,12$

( ا)

$u_{1}=u_{14}=\frac{n}{6}, u_{3}=u_{4}=\frac{2 n}{6}, n=0 \bmod 6$

$u_{i}=0 \quad i \neq 1,3,4,14$

(ii) $n=1 \bmod 2: G^{*}=\max _{u_{i}} G=\frac{1}{16}\left(11 n-\frac{3}{n}\right)$, the solutions satisfy the relations:

$u_{0}=u_{7}=u_{8}=u_{15}=0$,

$\left(u_{6}-u_{9}\right)-\left(u_{1}-u_{14}\right)= \pm 1$

$\left(u_{5}-u_{10}\right)-\left(u_{2}-u_{13}\right)= \pm 1$

$\left(u_{4}-u_{11}\right)-\left(u_{3}-u_{12}\right)=\mp 1$

$\left(u_{1}-u_{14}\right)+\left(u_{3}-u_{12}\right)+\left(u_{5}-u_{10}\right)=\mp 1$

$\left(u_{1}+u_{14}\right)-3\left(u_{2}+u_{13}\right)+2\left(u_{3}+u_{12}\right)-3\left(u_{4}+u_{11}\right)-6\left(u_{5}+u_{10}\right)-2\left(u_{6}+u_{9}\right)=0$

$u_{1}+u_{2}+u_{3}+u_{9}+u_{10}+u_{11}=\frac{n \mp 1}{2}$

Some solutions are:

$(\alpha)$

$u_{1}-1=u_{14}=u_{3}-2=u_{12}-2=u_{4}-1=u_{11}=\frac{n-7}{6}, u_{10}=1, n=1 \bmod 6$

$u_{i}=0 \quad i \neq 1,3,4,10,11,12,14, n \geq 7$

( $\beta)$

$u_{14}=2, u_{3}-2=u_{12}-1=u_{6}=u_{9}-1=u_{11}=\frac{n-7}{4}, u_{5}=1, n=3 \bmod 4$

$u_{i}=0 i \neq 3,5,6,9,11,12,14, n \geq 7$

$(\gamma)$

$u_{14}=2, u_{3}-2=u_{12}-1=u_{9}-1=u_{6}=\frac{n-7}{4}, u_{5}=1, n=3 \bmod 4, n \geq 7$

$u_{i}=0 \quad i \neq 3,5,6,9,12,14$

( $\delta)$

$u_{1}=1, u_{3}-2=u_{12}-2=u_{9}=u_{6}=\frac{n-7}{4}, u_{4}=u_{10}=1$,

$n=3 \bmod 4, n \geq 7, u_{i}=0 \quad i \neq 3,5,6,9,12,14$

$(\varepsilon)$

$u_{1}=1, u_{3}-2=u_{12}-2=u_{9}=u_{6}=\frac{n-7}{4}, u_{4}=u_{10}=1, n=3 \bmod 4, n \geq 7$

$u_{i}=0 i \neq 3,5,6,9,12,14$

$u_{1}-1=u_{14}-2=3 d, u_{2}=u_{13}-1=d, u_{3}-2=u_{12}-1=u_{9}=u_{6}=\frac{n-9}{4}-2 d$,

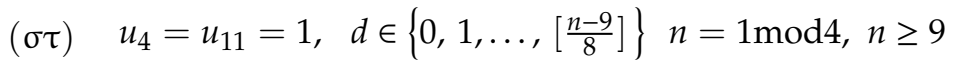

$u_{i}=0 \quad i \neq 1,2,3,4,6,9,11,12,13,14$

$u_{1}=u_{14}-1=3 d, u_{4}-2=u_{11}-2=d, u_{3}-4=u_{12}-3=u_{9}=u_{6}=\frac{n-13}{4}-2 d$,

(द) $u_{13}=1, d \in\left\{0,1, \ldots,\left[\frac{n-13}{8}\right]\right\} \quad n=1 \bmod 4, n \geq 13$

$u_{i}=0 \quad i \neq 1,3,4,6,9,11,12,13,14$

Proof. $n=0 \bmod 2$ : We look for the maximization of $G=\frac{1}{16}\left(R-q^{\mathbf{T}} \mathbf{M}^{-1} q\right)$, where $\mathbf{R}, \mathbf{q}, \mathbf{M}$ are of the form of (7). The maximum exists when $u_{0}+u_{7}+u_{8}+u_{15}=0$ and $\boldsymbol{q}=\mathbf{0}$. We have:

$$
\begin{array}{llll}
\left(u_{6}-u_{9}\right)-\left(u_{1}-u_{14}\right)=a & q_{1}=0 & a+b-c=0 & a=0 \\
\left(u_{5}-u_{10}\right)-\left(u_{2}-u_{13}\right)=b \Rightarrow & q_{2}=0 \\
\left(u_{4}-u_{11}\right)-\left(u_{3}-u_{12}\right)=c & q_{3}=0 & & -3 a-3 b+3 c=0
\end{array} \Leftrightarrow \begin{aligned}
& b=0 \\
& c=0
\end{aligned}
$$

The solutions $(\alpha)-(\iota)$ satisfy these necessary and sufficient conditions. $n=1 \mathrm{mod} 2$ : In that case $q_{i}=1 \bmod 2, i=1,2,3 \mathrm{ex}$. 


$$
\begin{aligned}
& q_{1}=-\left(u_{1}-u_{14}\right)-\left(u_{2}-u_{13}\right)+\left(u_{3}-u_{12}\right)-\left(u_{4}-u_{11}\right)+\left(u_{5}-u_{10}\right)+\left(u_{6}-u_{9}\right)= \\
& \left(u_{14}+u_{13}+u_{3}+u_{11}+u_{5}+u_{6}\right)-\left(u_{1}+u_{2}+u_{12}+u_{4}+u_{10}+u_{9}\right) \\
& =n-2\left(u_{1}+u_{2}+u_{12}+u_{4}+u_{10}+u_{9}\right)=1 \bmod 2
\end{aligned}
$$

Moreover:

$$
\mathbf{q}^{\mathbf{T}} \mathbf{M}^{-1} \mathbf{q}=\frac{1}{4 n}\left[q_{1}^{2}+q_{2}^{2}+q_{3}^{2}+\left(q_{1}+q_{2}+q_{3}\right)^{2}\right]+\frac{F^{2}}{D}
$$

with $F=\left[q_{4}-\frac{1}{4 n}\left[q_{1} m_{14}+q_{2} m_{24}+q_{3} m_{34}+\left(q_{1}+q_{2}+q_{3}\right)\left(m_{14}+m_{24}+m_{34}\right)\right]\right.$,

$D=m_{44}-\frac{1}{4 n}\left[m_{14}^{2}+m_{24}^{2}+m_{34}^{2}+\left(m_{14}+m_{24}+m_{34}\right)^{2}\right]>0$.

The quantities $q_{i}, i=1,2,3$ are odd and, $\mathbf{q}^{\mathbf{T}} \mathbf{M}^{-1} \mathbf{q} \geq \frac{1}{4 n}\left[q_{1}^{2}+q_{2}^{2}+q_{3}^{2}+\left(q_{1}+q_{2}+q_{3}\right)^{2}\right] \geq \frac{3}{n}$, so for example, $\left|q_{1}\right|=\left|q_{1}\right|=\left|q_{1}\right|=\left|q_{1}+q_{2}+q_{3}\right|=1$, does not give integer solutions for $\mathrm{a}, \mathrm{b}$, and $\mathrm{c}$.

The solutions which minimize $q_{1}^{2}+q_{2}^{2}+q_{3}^{2}+\left(q_{1}+q_{2}+q_{3}\right)^{2}$ are $q_{1}= \pm 3, q_{2}=\mp 1, q_{3}=\mp 1$, then $F=q_{4} \pm \frac{m_{14}}{n}$ and $q_{4}=0, m_{14}=0 \Rightarrow q^{T} M^{-1} q=\frac{3}{n}$.

So the conditions of maximization of $\mathrm{G}$ are,

$u_{0}+u_{7}+u_{8}+u_{15}=0, q_{1}= \pm 3, q_{2}=\mp 1, q_{3}=\mp 1$,

$q_{4}=0, m_{14}=0 \Rightarrow G=\frac{1}{16}\left(11 n-\frac{3}{n}\right) \Rightarrow \operatorname{var}\left(\hat{\delta}_{\mathrm{A}}-\hat{\delta}_{\mathrm{B}}\right)=\sigma^{2} \frac{16 n}{11 n^{2}-3}$.

The $(\alpha)-(\zeta)$ satisfy these conditions.

\section{Discussion}

In the case of four periods, carry over effects optimization (in all the cases) includes the designs where $u_{0}=u_{7}=u_{8}=u_{15}=0$, (all the treatments are the same) as in the case of direct effects. In this case, it is obvious that there are many more solutions from the cases of two and three periods [11,13]. The cases with a greater number of periods and the examination for the case of the model with interactions periods - either for carry over effects or for direct effects-are of interest.

Funding: This research received no external funding.

Conflicts of Interest: The authors declare no conflict of interest.

\section{Appendix A}

$$
\begin{gathered}
X_{10}=X_{18}=\left[\begin{array}{l}
0 \\
1 \\
1 \\
1
\end{array}\right], X_{11}=X_{19}=\left[\begin{array}{c}
0 \\
-1 \\
1 \\
1
\end{array}\right], X_{12}=X_{1,10}=\left[\begin{array}{c}
0 \\
1 \\
-1 \\
1
\end{array}\right], X_{13}=X_{1,11}=\left[\begin{array}{c}
0 \\
-1 \\
-1 \\
1
\end{array}\right], \\
X_{14}=X_{1,12}=\left[\begin{array}{c}
0 \\
-1 \\
1 \\
-1
\end{array}\right], X_{15}=X_{1,13}=\left[\begin{array}{c}
0 \\
-1 \\
1 \\
-1
\end{array}\right], X_{16}=X_{1,14}=\left[\begin{array}{c}
0 \\
1 \\
-1 \\
-1
\end{array}\right], X_{17}=X_{1,15}=\left[\begin{array}{cccc}
\pi_{1} & \pi_{2} & \pi_{3} & \tau \\
-1 \\
-1 \\
-1
\end{array}\right], \\
\mathbf{X}_{20}=\left[\begin{array}{llll}
1 & 0 & 0 & 1 \\
0 & 1 & 0 & 1 \\
0 & 0 & 1 & 1 \\
0 & 0 & 0 & 1
\end{array}\right] \quad \mathbf{x}_{22}=\left[\begin{array}{cccc}
0 & 1 & 0 & -1 \\
0 & 0 & 1 & 1 \\
0 & 0 & 0 & 1
\end{array}\right] \\
\mathbf{X}_{21}=\left[\begin{array}{llll}
1 & 0 & 0 & -1 \\
0 & 1 & 0 & 1 \\
0 & 0 & 1 & 1 \\
0 & 0 & 0 & 1
\end{array}\right] \mathbf{X}_{23}=\left[\begin{array}{cccc}
1 & 0 & 0 & -1 \\
0 & 1 & 0 & -1 \\
0 & 0 & 1 & 1 \\
0 & 0 & 0 & 1
\end{array}\right],
\end{gathered}
$$




$$
\begin{aligned}
& \mathbf{X}_{24}=\left[\begin{array}{cccc}
\mu \pi_{1} & \pi_{2} & \pi_{3} \tau \\
1 & 0 & 0 & 1 \\
0 & 1 & 0 & 1 \\
0 & 0 & 1 & -1 \\
0 & 0 & 0 & 1
\end{array}\right] \quad \mathbf{X}_{25}=\left[\begin{array}{cccc}
1 & 0 & 0 & 1 \\
0 & 1 & 0 & -1 \\
0 & 0 & 1 & 1 \\
0 & 0 & 0 & 1
\end{array}\right] \\
& \mathbf{X}_{26}=\left[\begin{array}{cccc}
1 & 0 & 0 & 1 \\
0 & 1 & 0 & -1 \\
0 & 0 & 1 & -1 \\
0 & 0 & 0 & 1
\end{array}\right] \quad \mathbf{X}_{27}=\left[\begin{array}{cccc}
1 & 0 & 0 & -1 \\
0 & 1 & 0 & -1 \\
0 & 0 & 1 & -1 \\
0 & 0 & 0 & 1
\end{array}\right],
\end{aligned}
$$

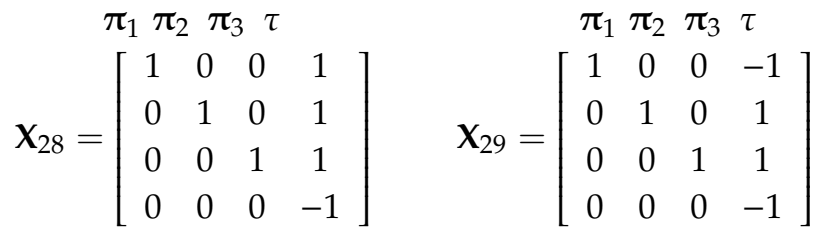

$$
\begin{aligned}
& \mathbf{X}_{210}=\left[\begin{array}{cccc}
1 & 0 & 0 & 1 \\
0 & 1 & 0 & -1 \\
0 & 0 & 1 & 1 \\
0 & 0 & 0 & -1
\end{array}\right] \quad \mathbf{X}_{2,11}=\left[\begin{array}{cccc}
1 & 0 & 0 & -1 \\
0 & 1 & 0 & -1 \\
0 & 0 & 1 & 1 \\
0 & 0 & 0 & -1
\end{array}\right] \text {, } \\
& \begin{array}{llllllll}
\pi_{1} & \pi_{2} & \pi_{3} & \tau & \pi_{1} & \pi_{2} & \pi_{3} & \tau
\end{array} \\
& \mathbf{X}_{2,12}=\left[\begin{array}{cccc}
1 & 0 & 0 & 1 \\
0 & 1 & 0 & 1 \\
0 & 0 & 1 & -1 \\
0 & 0 & 0 & -1
\end{array}\right] \quad \mathbf{X}_{2,13}=\left[\begin{array}{cccc}
1 & 0 & 0 & -1 \\
0 & 1 & 0 & 1 \\
0 & 0 & 1 & -1 \\
0 & 0 & 0 & -1
\end{array}\right] \\
& \mathbf{X}_{21}=\left[\begin{array}{cccc}
1 & 0 & 0 & 1 \\
0 & 1 & 0 & -1 \\
0 & 0 & 1 & -1 \\
0 & 0 & 0 & -1
\end{array}\right] \quad \mathbf{X}_{23}=\left[\begin{array}{llll}
1 & 0 & 0 & -1 \\
0 & 1 & 0 & -1 \\
0 & 0 & 1 & -1 \\
0 & 0 & 0 & -1
\end{array}\right]
\end{aligned}
$$

\section{References}

1. Lawes, J.B.; Gilbert, J.H. Report of experiments on the growth of wheat for twenty years in succession on the same land. J. Agric. Soc. Engl. 1864, 25, 449-451.

2. Chalikias, M.; Kounias, S. Extension and necessity of Cheng and Wu conditions. J. Stat. Plan. Infer. 2012, 142, 1794-1800. [CrossRef]

3. Hedayat, A.S.; Yang, M. Universal Optimality of Selected Crossover Designs. J. Am. Stat. Assoc. 2004, 99, 461-466. [CrossRef]

4. Hedayat, A.S.; Zheng, W. Optimal and efficient crossover designs for test-control study when subject effects are random. J. Am. Stat. Assoc. 2010, 105, 1581-1592. [CrossRef]

5. Stufken, J. Some families of optimal and efficient repeated measurements designs. J. Stat. Plan. Infer. 1991, 27, 75-83. [CrossRef]

6. Hedayat, A.S.; Yan, Z. Crossover designs based on type I orthogonal arrays for a self and simple mixed carryover effects model with correlated errors. J. Stat. Plan. Infer. 2008, 138, 2201-2213. [CrossRef]

7. Kounias, S.; Chalikias, M.S. An algorithm applied to designs of repeated measurements. J. Appl. Stat. Sci. 2005, 14, 243-250.

8. Kushner, H.B. Allocation rules for adaptive repeated measurements designs. J. Stat. Plan. Infer. 2003, 113, 293-313. [CrossRef]

9. Laska, E.M.; Meisner, M. A variational approach to optimal two treatment crossover designs: Application to carryover effect models. J. Am. Stat. Assoc. 1985, 80, 704-710. [CrossRef]

10. Hedayat, A.; Afsarinejad, K. Repeated measurements designs, I. In A Survey of Statistical Design and Linear Models; Journal of the Royal Statistical Society: New York, NY, USA, 1975; pp. 229-242. 
11. Kounias, S.; Chalikias, M.; Vonta, F. Optimal and Universally Optimal Two Treatment Repeated Measurement Designs; Vonta, F., Nikulin, M., Eds.; Statistics for industry and technology Birkhauser: Boston, MA, USA; Basel, Switzerland; Berlin, Germany, 2008; pp. 465-477.

12. Chalikias, M.; Kounias, S. Optimal two Treatment Repeated Measurement Designs for three Periods. Commun. Stat. Theory Methods 2017, 46, 200-209. [CrossRef]

13. Kounias, S.; Chalikias, M.S. Optimal two treatment repeated measurement designs with treatment-period interaction in the model. Util. Math. 2015, 96, 243-261.

14. Kounias, S.; Chalikias, M. Optimal cross over designs of two treatments and four periods the case of direct effects. In Proceedings of the 18th Conference of Greek Statistical Institute, Rhodes, Greece, 12-16 September 2005; pp. 201-209. (In Greek).

15. Hedayat, A.S.; Afsarinejad, K. Repeated measurements designs II. Ann. Stat. 1978, 18, 1805-1816. [CrossRef]

16. Chalikias, M.S. Optimal Repeated Measurements for Two Treatment Designs with Dependent Observations: The Case of Compound Symmetry. Mathematics 2019, 7, 378. [CrossRef]

17. Kounias, S.; Chalikias, M. Estimability of Parameters in a Linear Model. Stat. Probab. Lett. 2008, 28, $2437-2439$. [CrossRef]

(C) 2019 by the author. Licensee MDPI, Basel, Switzerland. This article is an open access article distributed under the terms and conditions of the Creative Commons Attribution (CC BY) license (http://creativecommons.org/licenses/by/4.0/). 